\title{
Ruthenium Red Staining Reveals Surface Fibrils and a Layer External to the Cell Wall in Streptococcus salivarius HB and Adhesion Deficient Mutants
}

\author{
By PAULINE S. HANDLEY, * JUNE HARGREAVES AND \\ DEREK W. S. HARTY, \\ Department of Cell and Structural Biology, Stopford Building, Manchester University, \\ Oxford Road, Manchester M13 9PT, UK
}

(Received 6 June 1988)

\begin{abstract}
Ruthenium red staining revealed both the long and short classes of cell surface fibril in thin sections of Streptococcus salivarius HB, indicating that the fibrils contained polyanionic polymers, probably polysaccharides. Also visible was a $16.2 \pm 2.2 \mathrm{~nm}$ thick ruthenium red staining layer (RRL) outside the $16.7 \pm 2.2 \mathrm{~nm}$ thick cell wall. The fibrils could not be seen after conventional glutaraldehyde and osmium fixation. The $R R L$ was protease resistant and was not involved in septum formation. Loss of the fibrils after protease treatment coincided with a decrease of $54 \%$ in cell surface hydrophobicity, indicating that cell surface hydrophobicity was due partly to fibrils and partly to the RRL. There was no correlation between the lengths of fibrils as measured on whole cells after negative staining and on thin sections of ruthenium red stained cells. The thickness of the RRL was the same in three adhesion deficient mutants strains HB-7, HB-V5 and HB-V51 - with various fibril lengths. However, a completely bald mutant, HB-B, had a significantly thicker RRL than $S$. salivarius HB, although it was unable to adhere to buccal epithelial cells, and it could not co-aggregate with Veillonella paroula V1. The RRL therefore did not contain adhesins.
\end{abstract}

\section{INTRODUCTION}

The cell surface of Streptococcus salivarius carries a number of structurally and functionally distinct classes of fibrils, peritrichously distributed over the cell. Ultrastructural analysis of adhesion deficient mutants, using negative staining, has shown that strain HB carries long $(178 \mathrm{~nm})$ protease resistant fibrils, and three classes of shorter, protease sensitive fibrils $-91,72$ and $63 \mathrm{~nm}$ long respectively (Handley et al., 1984; Weerkamp et al., 1986). The $91 \mathrm{~nm}$ fibrils (antigen B) are responsible for co-aggregation with Veillonella parvula V1, and the $72 \mathrm{~nm}$ fibrils (antigen C) are responsible for adhesion to host surfaces. The functions of the $178 \mathrm{~nm}$ and $63 \mathrm{~nm}$ fibrils are unknown (Weerkamp et al., 1986).

Previously, we studied adhesion deficient mutants of $S$. salivarius HB by ultrastructural analysis of methylamine tungstate stained preparations (Weerkamp et al., 1986). This technique of negative staining is considered to give a reproducible image with excellent resolution, within the limits presented by biological specimens. However, negative staining does not reveal details of the underlying structure of the surface layers and gives no information on their chemical composition.

Ruthenium red staining has been widely used in electron microscopy to locate acidic polysaccharide like material. The technique was studied in detail by Luft (1971) and used in a number of subsequent studies on surface structure and composition, often describing adhesive components (Costerton \& Irvin, 1981 ; Fletcher \& Floodgate, 1973; Brooker, 1979; Jones et al.,

Abbreviations: RRL, Ruthenium red staining layer; BEC, buccal epithelial cells; TA, teichoic acid; LTA, lipoteichoic acid. 
1969). Ruthenium red complexes in a catalytic reaction with carboxyl or hydroxyl groups in the presence of osmium tetroxide to give an electron dense precipitate. Polymers carrying a high charge density, usually acidic polysaccharides, are stained in this way (Luft, 1971). Brooker (1979) also reported that the neutral polysaccharide dextran, on the surface of Leuconostoc mesenteroides, is also stained by ruthenium red. Polysaccharides are not detected after conventional fixation. When $S$. salivarius cells were fixed in osmium and glutaraldehyde and post-stained (Gibbons et al., 1972), they were found to carry a fairly thin amorphous layer called a 'fuzzy coat' outside the cell wall, with only a suggestion of fibrils radiating outwards from the cell wall. Structures equivalent to clearly defined fibrils seen by negative staining (Handley $e t a l$., 1984) were not seen.

The work reported in this paper describes the surface ultrastructure of $S$. salivarius $\mathrm{HB}$ and four adhesion deficient mutants, after ruthenium red staining.

\section{METHODS}

Organisms and culture methods. S. salivarius HB and the mutant strains HB-7, HB-V5 and HB-V51 were kindly provided by Dr A. H. Weerkamp (University of Groningen, The Netherlands). Strain HB carries three different lengths of fibril - $178 \mathrm{~nm}$ (unknown function), $91 \mathrm{~nm}$ (antigen B or co-aggregation adhesin) and $73 \mathrm{~nm}$ (antigen C or host associated adhesion factor). Strain HB-7 carries only the $91 \mathrm{~nm}$ fibrils, HB-V5 carries the $178 \mathrm{~nm}$ and $73 \mathrm{~nm}$ fibrils and strain HB-V51 carries $63 \mathrm{~nm}$ fibrils of unknown function. These measurements had been established by us previously (Weerkamp et al., 1986). Strain HB-B was a spontaneous variant which occurred in a chemostat culture grown under glucose limitation at a slow dilution rate $\left(0-148 \mathrm{~h}^{-1}\right)$. It had lost the ability to adhere to buccal epithelial cells (BEC) and could not co-aggregate with $V$. paroula V1.

The strains were stored at $-70^{\circ} \mathrm{C}$ in glycerol and were grown up for each experiment on Brain Heart Infusion agar (BHI) with $5 \%(\mathrm{v} / \mathrm{v})$ horse blood. Liquid cultures were then grown in BHI broth with $0.3 \%$ yeast extract for $18 \mathrm{~h}$ at $37^{\circ} \mathrm{C}$ in a candle jar. (A jar in which a candle has been burned until extinguished in order to lower the amount of oxygen available.)

Ruthenium red staining. A modification of the method used by Luft (1971) was used. A solution of ruthenium red (Johnson \& Matthey Chemicals) was made up in water to a final concentration of $10 \mathrm{mg} \mathrm{m}^{-1}$ (1500 p.p.m.). Treated or untreated cells were washed three times in $0.2 \mathrm{M}$-sodium cacodylate buffer (pH 7.3) and fixed initially in

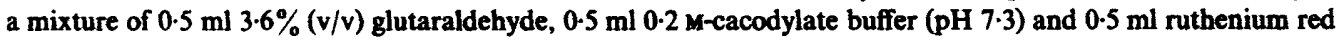
stock solution for $1 \mathrm{~h}$ at room temperature. After three washings in $\mathbf{0 . 2} \mathrm{M}$-cacodylate buffer, cells were fixed in a mixture of $0.5 \mathrm{ml} 4 \%(\mathrm{w} / \mathrm{v})$ osmium tetroxide in distilled water, $0.5 \mathrm{ml} \mathrm{0.2} \mathrm{M-cacodylate} \mathrm{buffer} \mathrm{(pH} \mathrm{7.3)} \mathrm{and} 0.5 \mathrm{ml}$ ruthenium red stock solution. The cells were fixed overnight in this mixture, as the intensity of the ruthenium red staining was found to be markedly greater than when fixed for $3 \mathrm{~h}$ as recommended by Luft (1971). Controls were prepared by substituting distilled water for the ruthenium red stock solution. Cells were then washed three times in 0.2 M-cacodylate buffer, dehydrated in a graded series of ethanol and embedded in either Spurr resin (Agar Aids) or LR white resin (London Resin Co.). Sections were cut on a Reichert OMU4 microtome and sections were photographed on a Hitachi H600 electron microscope.

Cell surface hydrophobicity. The method used by Rosenberg et al. (1980) was modified as reported by Handley et al. (1987). Briefly, cells were washed three times in $0.066 \mathrm{~m}$ Sörensons phosphate buffer (pH 7.2) and resuspended to an $\mathrm{OD}_{440}$ of 0.5 . Samples of cells $(3.0 \mathrm{ml})$ were blended for $60 \mathrm{~s}$ on a vortex mixer after $200 \mu$ l hexadecane (BDH) had been layered on top. The tubes were left to stand for $15 \mathrm{~min}$ for phase separation to occur and the $\mathrm{OD}_{440}$ of the aqueous phase was measured. Hydrophobicity was calculated from six replicates as the percentage decrease in optical density of the original bacterial suspension due to cells partitioning into the hexadecane layer (Handley et al., 1987).

Protease treatment. S. salivarius strains were grown in BHI broth plus $0.3 \%$ yeast extract overnight and washed in water three times. Protease treatment (protease type XIV from Streptomyces griseus; Sigma) was done as described by Handley $e t$ al. (1984) at a final concentration of $0.5 \mathrm{mg} \mathrm{ml}^{-1}$ in Sörensons phosphate buffer at pH 7.2.

Measurements. The width of the cell wall and ruthenium red staining layer (RRL) were all measured on micrographs at a magnification of $\times 114000$. Thin pen lines were drawn at the internal and external perimeters of the two layers and the distances between them measured with a graticule divided into tenths of a millimetre. When 20 identical micrographs were measured for cell wall thickness, the measurement error was found to be $\pm 0.8 \mathrm{~nm}$. The widths of the fibrillar layers after ruthenium red staining were measured from a line drawn around the outer perimeter of the fibrils to a line drawn at the base of the RRL (see Results). All mean measurements are quoted with the standard deviations, which includes the measurement error. All measurements from ruthenium red stained sections were made on $\mathbf{3 0}$ different micrographs. The measurements of fibril lengths after negative staining were made on 10 different micrographs (Weerkamp et al., 1986). 
Statistics. The relationship of the thickness of both the wall and the RRL within batch measurements, between batch measurements and between strains, was analysed using the nested analysis of variance design (Winer, 1971). To test for differences between strains the variance ratio of variability between batches to variability between strains was calculated (Handley et al., 1987).

\section{RESULTS}

\section{Ruthenium red staining of $S$. salivarius $H B$ and its adhesion deficient mutants}

Fibrils on the cell surface of $S$. salivarius HB were revealed in thin sections by ruthenium red staining as a dense confluent layer of short fibrils with longer fibrils projecting through (Fig. $1 a$ ). At the base of the fibrillar fringe a more electron dense RRL was seen. This was consistently found on every cell of all strains examined. The fibrils differed in density from cell to cell in a population, sometimes revealing just the wall and the RRL, with patches carrying no fibrils, but with an intact RRL entirely covering the cells (micrographs not presented). Mutants HB-V5 (Fig. 1b), HB-7 (Fig. 1 c), HB-V51 (Fig. 2a) and HB-B (Fig. 2b) also carried the RRL outside the cell wall on all cells. The RRL had a loose amorphous outer edge which could be seen more clearly when few or no fibrils were present on a strain (Figs $1 c$ and $2 a, b, d$ ). In order to look for possible differences between mutants, other than fibril length, the thickness of the RRL was measured for all strains. Two batches of each strain were grown simultaneously in BHI broth with $0.3 \%$ yeast extract and stained with ruthenium red, before thin sectioning all at the same time. Samples were also negatively stained. Cells were embedded in LR white resin.

The thickness of the RRL was measured on 30 micrographs for each strain. Statistical analysis showed that the RRL on HB-B $(18.0 \pm 2.2 \mathrm{~nm})$ was significantly thicker $(P=0.000018)$ than the RRL $(16 \cdot 2 \pm 2 \cdot 2 \mathrm{~nm})$ on HB. This was true for both batches of cells. There was no significant batch to batch variation in the RRL thickness of either strain, and no other differences were found in RRL thickness when statistical comparisons were made between $H B$ and HB-7 and HB-V51. HB-V51 RRL thickness was compared to that of HB-V5 RRL, the strain from which it was derived (Weerkamp et al., 1987). The mean wall thickness for strain HB was $16.7 \pm 2.2 \mathrm{~nm}$ and there were no significant differences between wall thickness when the mutants were compared with HB ( 30 micrographs from each of two batches were measured).

When the lengths of fibrils were compared after negative staining and after ruthenium red staining no consistent correlations emerged. Lengths of fibrils in ruthenium red stained preparations were measured from the end of the fibrils to the base of the RRL. Fig. $1(d)$ is a negatively stained preparation of HB-7 showing that the cell surface is smooth; this was so for all cells in all strains. In contrast, the outer surface of the RRI is very uneven (Figs $1 c$ and $2 a, b$, $d)$ : therefore it is thus probable that negative staining with methylamine tungstate penetrates through the RRL. In addition, polysaccharides may not be stainable with negative stains as they lack the necessary bulky side chains of amino acids for exclusion of the stain (Tzaphlidou \& Chapman, 1986). The perimeter seen after negative staining is therefore probably the surface of the cell wall itself.

After ruthenium red staining the short fibrillar fringe on strain $\mathrm{HB}$ was found to have shrunk by $22 \%$ when compared to measurements taken on fibril length after negative staining on the same batch of cells grown in BHI broth for $18 \mathrm{~h}$ (Table 1). This shrinkage factor was consistent, and similar values were obtained in three separate experiments (three growth batches, each stained and fixed separately) on strain HB. In contrast no shrinkage occurred in the fibrillar fringe of HB-7 or HB-V5 as the lengthe of the short fibrils after ruthenium red staining were similar to the measurements on the same batch of cells after negative staining (Table 1). The fibrillar fringe of HB-V 51 was $12 \%$ longer by ruthenium red staining than by negative staining (Table 1). The reasons for shrinkage and apparent extension after ruthenium red staining are not known.

The RRL was not involved in septum formation (Fig. $2 d$ ) and only appeared on the surface of the new wall after the cells had separated. This was observed for all cells in all strains.

When $S$. salivarius HB was fixed in glutaraldehyde and osmium and post-stained with uranyl acetate and lead citrate, a discontinuous, very loose layer, with a variable width, was observed 


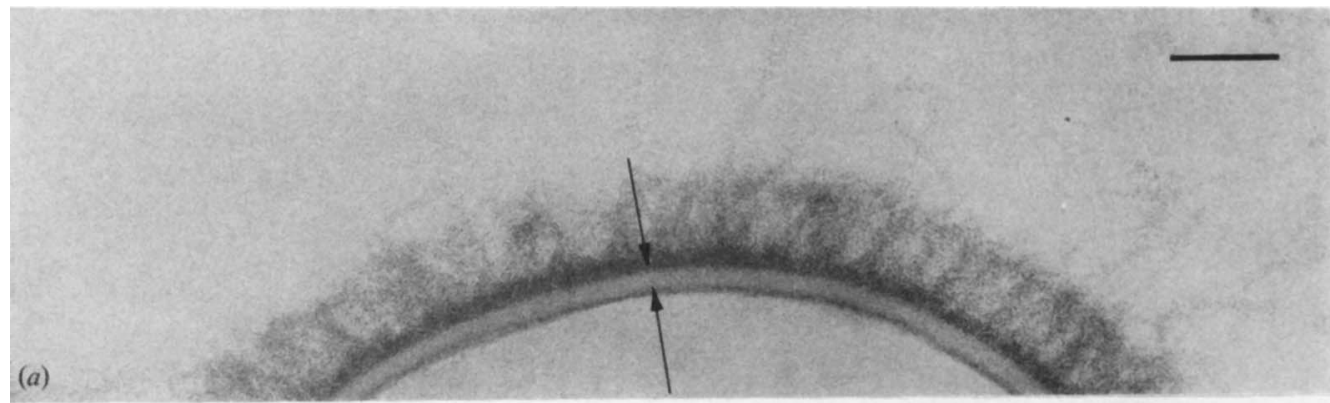

(b)
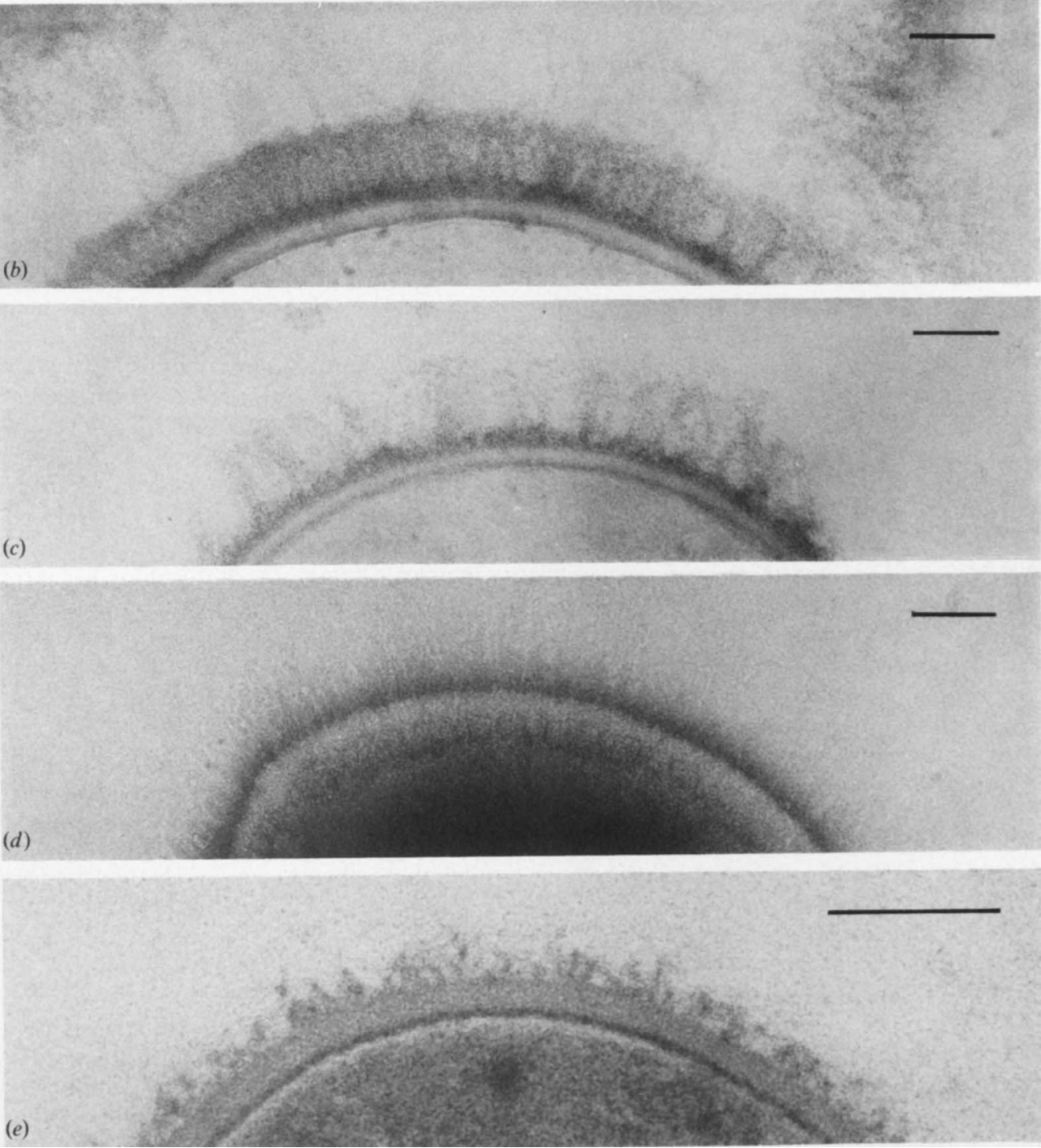

Fig. 1. The sectioned cells in $(a)-(c)$ were all stained with ruthenium red, but were not post-stained. (a) Strain HB showing long fibrils projecting through the more densely distributed short fibrils with the electron dense RRL underneath. Arrows indicate wall thickness. (b) HB-V5 also shows long fibrils projecting through a dense fringe of shorter fibrils. (c) HB-7 carries sparse short fibrils only, with the RRL underneath, outside the cell wall. (d) HB-7 negatively stained with $1 \%$ methylamine tungstate. (e) A section of strain $\mathrm{HB}$ after conventional fixation in glutaraldehyde and osmium, and post-staining with uranyl acetate and lead citrate, showing a loose, discontinuous layer outside the cell wall. Bar markers represent $100 \mathrm{~nm}$. 

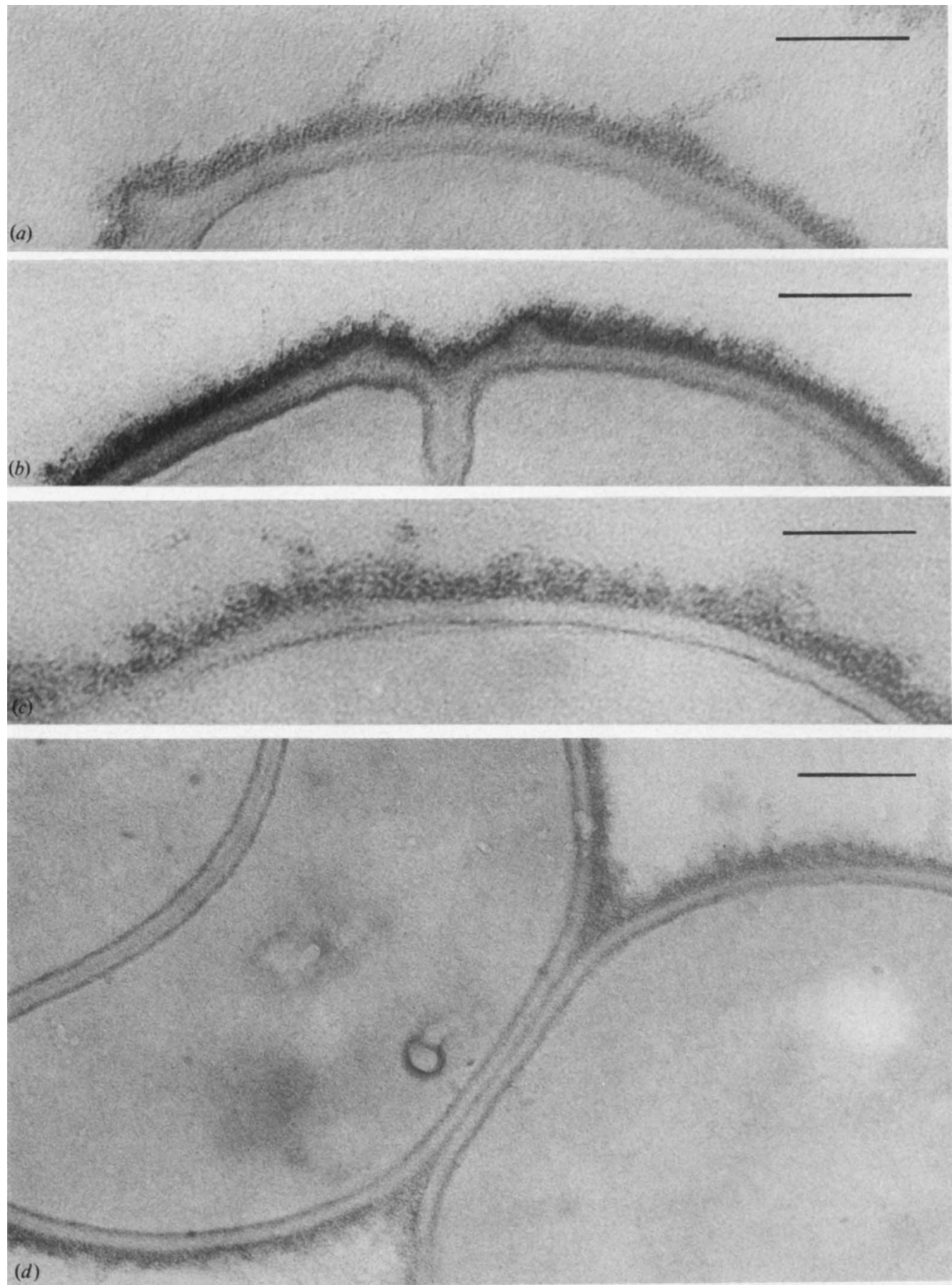

Fig. 2. All sectioned cells wete stained with ruthenium red, but were not post-stained. (a) Strain HBV51 showing very sparse fibrils attached to the continuous RRL outside the cell wall. (b) HB-B has no fibrils, but carries a RRL. (c) HB after protease treatment showing a RRL with a loosened edge. (d) HB7 showing two septa. The RRL is not involved with septum formation and only occurs at the cell surface. Bars represent $100 \mathrm{~nm}$. 
Table 1. Comparison of the lengths of the short fibrils after negative staining and ruthenium red staining

Measurements after ruthenium red staining were done on micrographs of thin sections; measurements after negative staining were done on micrographs of whole cells.

\begin{tabular}{lcc} 
& \multicolumn{2}{c}{ Length (nm) } \\
\cline { 2 - 3 } Strain & Ruthenium red staining* & Negative stainingt \\
HB & $68.0 \pm 6.9$ & $87.1 \pm 5.0$ \\
HB-V5 & $74.9 \pm 3.6$ & $73.6 \pm 10.7$ \\
HB-7 & $91.3 \pm 7.7$ & $90.0 \pm 3.0$ \\
HB-V51 & $73.2 \pm 4.9$ & $64.4 \pm 4.6$
\end{tabular}

* Measurements represent the mean of the fibril length measured on 30 separate micrographs, all from one batch of cells. Measurements were taken from the end of the fibrils to the base of the RRL.

$\dagger$ Measurements taken on 10 separate micrographs of the same batch of cells as used for ruthenium red staining.

outside the cell wall of all cells in a population (Fig. $1 e$ ). The cell wall thickness $(16.2 \pm i \cdot 6 \mathrm{~nm}$; mean of two batches of 10 replicate measurements) was not significantly different from the cell wall thickness measured after ruthenium red staining.

\section{Protease treatment and hydrophobicity measurements}

S. salivarius $\mathrm{HB}$ was treated with $0.5 \mathrm{mg}$ protease $\mathrm{ml}^{-1}$ at $37^{\circ} \mathrm{C}$ for up to $3 \mathrm{~h}$. Samples of cells were simultaneously negatively stained, fixed and stained with ruthenium red and tested for changes in hydrophobicity. We previously reported that the short fibrils are protease sensitive and the long fibrils are protease resistant after $2 \mathrm{~h}$ treatment at this concentration (Handley $e t$ al., 1984). However, after $3 \mathrm{~h}$ treatment the long fibrils were removed, intact, from the cell surface by the longer exposure to protease. Fig. 2(c) shows the surface of strain HB after $3 \mathrm{~h}$ of protease treatment, still with an intact RRL. All protease treated cells carried a RRL which at $17.3 \pm 1.3 \mathrm{~nm}$ was not significantly different from the thickness of the RRL before protease treatment $(16.2 \pm 2.2 \mathrm{~nm})$. The edge of the RRL appeared to be slightly looser after protease treatment (Fig. $2 c$ ) which would account for the slightly thicker measurement for the RRL.

The cell surface hydrophobicity of strain $\mathrm{HB}$ before protease treatment was $94.2 \pm 1.5 \%$, but after treatment it dropped to $43.4 \pm 5 \cdot 3 \%$ (mean of two batches, six replicates each), a drop of $54 \%$.

\section{Chemostat variant $H B-B$}

$S$. salivarius strain HB-B arose spontaneously in a glucose limited chemostat after prolonged growth of strain $\mathrm{HB}$ at a low dilution rate $\left(0.148 \mathrm{~h}^{-1}\right)$. This mutant was recognized by its inability to adhere to BEC and to co-aggregate with $V$. parvula $V 1$. In addition, it had a very low cell surface hydrophobicity $(27.9 \pm 6.8 \%$ ) after growth in BHI for $18 \mathrm{~h}$ as compared to $\mathrm{HB}$ $(94-2 \pm 1.5)$ a difference of $70.4 \%$. Similar variants often appeared at low growth rates. HB-B was completely bald by negative staining, and carried an RRL outside the wall. However, the RRL was significantly thicker than that of $\mathrm{HB}$.

\section{DISCUSSION}

Fixing and ruthenium red staining of $S$. salivarius $\mathrm{HB}$ and its mutants preserved both long and short fibril classes and revealed a homogeneous layer outside the cell wall which is the same thickness as the wall itself. The mean value for the wall thickness after ruthenium red staining was $16.7 \pm 2.2 \mathrm{~nm}$, which is quite thin. The thinnest wall so far reported for a Gram-positive organism is $15.17 \mathrm{~nm}$ for Butyrivibrio fibrisolvens (Cheng \& Costerton, 1977). The B. fibrisolvens wall thickness was quoted after glutaraldehyde and osmium fixation and post-staining. The ruthenium red stained sections in this study were not post-stained to maximize the contrast produced by ruthenium red, but they were fixed in glutaraldehyde and osmium. Ruthenium red 
staining did not itself alter the wall thickness. (It should be noted that the wall layer was measured - see arrows in Fig. $1 a$ - between the inner edge of the RRL and an electron dense band, which was assumed to belong to the plasma membrane.)

The outer edge of the RRL was not as well defined as the edge of the wall. Although it is electron dense, the RRL had a looser, less homogeneous appearance than the cell wall. The fuzzy coat previously described by Gibbons et al. (1972) on $S$. salivarius is the layer outside the cell wall which can be seen after conventional osmium and glutaraldehyde fixation. The fuzzy coat was also seen in this study in the absence of ruthenium red and it appeared as a very loose, patchy fibrous layer (Fig. 1e) with an indeterminate width. Glutaraldehyde fixes proteins and osmium tetroxide fixes primarily lipids, so any polysaccharide outside the cell wall would not be detected by conventional glutaraldehyde and osmium fixation. Ruthenium red is therefore revealing components not seen by conventional fixation.

The fibrils are likely to be different in composition from the RRL as protease removes both short and long fibrils, leaving the RRL intact, although with an apparently loosened edge. The fibrils consist of glycoproteins (Weerkamp \& Jacobs, 1982), and antigens B and C have different relative proportions of protein, hexoses and amino sugars. Ruthenium red is probably staining the polysaccharide component present in the fibrils, although this is predominantly in the form of neutral sugar (Weerkamp \& Jacobs, 1982).

It is not yet known whether the RRL should be considered to be part of the cell wall. It is not involved with septum formation and remains at the cell surface as the septum grows inwards. However, the outer membrane of Gram-negative cells is thought not to be involved with septum formation (Burdett \& Murray, 1974) and yet the outer membrane is considered to be part of the cell wall. The cell walls of Gram-positive bacteria are consistently described as homogeneous layers, containing peptidoglycan and teichoic acid (TA), usually ranging from 20-50 nm thick (Shockman \& Barrett, 1983), although the exact location of TA is not clear. Polysaccharide containing layers have not been previously observed outside the cell wall, presumably because ruthenium red staining has not been included in the fixation procedures. We have found that a RRL is common in oral streptococci, and $S$. sanguis strain 311 , which has a lateral tuft of fibrils, also carries a thick $37 \pm 6 \mathrm{~nm}$ RRL layer (Hesketh et al., 1987). Strains of $S$. milleri and $S$. salivarius (a Lancefield $K$ - strain) with peritrichous fimbriae, and $S$. sanguis strains with peritrichous fibrils, also carry a similar layer outside the wall (P. S. Handley unpublished data). Brooker (1979) found that different $L$. mesenteroides strains show a variety of different, very complex, surface layer patterns, outside the cell wall after ruthenium red staining, and that growth in different media influences the appearance of the ruthenium red staining layers. It is therefore possible that surface layers containing polymers with a high charge density (usually polysaccharides) are more common than previously thought on the surfaces of Gram-positive bacteria.

In support of this suggestion are the many biochemical observations showing that neutral and acidic polysaccharides covalently linked to the peptidoglycan are common in Gram-positive bacteria (Shockman \& Barrett, 1983; Rogers et al., 1980) and are considered to be components of the cell wall. For example, in Group B streptococci both the Group B polysaccharide and the type III spocific polysaccharide are covalently bound to the peptidoglycan and are considered to be part of the cell wall (de Cueninck et al., 1982). If the RRL of $S$. salivarius is found to be covalently bound to peptidoglycan then it may be considered as a structural wall component.

The composition of the $S$. salivarius $R R L$ is unknown, although some surface polysaccharides have been identified in other studies. The Lancefield Group $\mathbf{K}$ cell wall polysaccharide antigen of $S$. salivarius has been isolated and contains galactose, glucose, rhamnose and a trace of glucosamine (Montague \& Knox, 1968). The structural location of the Group $\mathrm{K}$ antigen is unknown, but it may be located in the RRL, because strains HB-V5, HB-V51 and HB-7 all produce the Group $K$ antigen (Weerkamp et al., 1987) and they all carry the RRL. It is not known whether HB-B or protease treated cells still carry the Group $K$ antigen.

Lipoteichoic acid (LTA), a negatively charged polymer of polyglycerophosphate is present in $S$. salivarius $\mathrm{HB}$ and LTA exposure at the cell surface is inversely related to the fibril density. In another bald strain, HB-C12, more LTA was exposed than in strain HB (Weerkamp et al., 1987). 
This observation indicates that LTA may be present in the RRL.

It is not known why the RRL of strain HB-B is thicker than the same layer for HB. Preliminary studies on the RRL thickness for HB-B grown at different dilution rates in a glucose limited chemostat show that the thickness of this layer varies with growth rate, a variation not shown by HB. The RRL is therefore capable of phenotypic changes. Whether biochemical changes coincide with structural changes is presently under study.

We gratefully acknowledge the receipt of a grant from the Medical Research Council (no. 8506176) for D.W.S.H.

\section{REFERENCES}

BrooksR, B. E. (1979). Electron microscopy of the dextrans produced by lactic acid bacteria. In Microbial Polysaccharides and Polysaccharases, Special Publication no. 3 of the Society for General Microbiology, pp. 85-115. Edited by R. C. W. Berkeley, G. W. Gooday \& D. C. Ellwood. London: Academic Press.

BURDETT, I. D. J. \& MURRAY, R. G. E. (1974). Septum formation in Escherichia coli: characterization of septal structure and the effects of antibiotics on cell division. Journal of Bacteriology 119, 303-324

Cheng, K.-J. \& Costerton J. W. (1977). Ultrastructure of Butyrivibrio fibrisolvens: a Gram-positive bacterium? Journal of Bacteriology 129, 1506-1512.

Costerton, J. W. IRvin, R. T. (1981). The bacterial glycocalyx in nature and disease. Anmual Review of Microbiology 35, 299-324.

De Cueninck, B. J., Shockman, G. D. \& Ward, J. B. (1980). Group B, type III streptococcal cell wall: composition and structural aspects revealed through endo-N-acetyl muramidase-catalysed hydrolysis. Infection and Immonity 35, 572-582.

Gibdons, R. J., van Houtr, J. \& LILJEMark, W. F. (1972). Parameters that effect the adherence of Streptococcus salivarius to oral epithelial surfaces. Journal of Dental Research 51, 424-435.

Fletcher, M. \& FlOODGATE, G. D. (1973). An electron microscopic demonstration of an acidic polysaccharide involved in the adhesion of a marine bacterium to solid surfaces. Journal of General Microbiology 74, 325-334.

Handley, P. S., Carter, P. L. \& Fiblding, J. (1984). Streptococcus salivarius strains carry either fibrils or fimbriae on the cell surface. Journal of Bacteriology 157, 64-72.

HANDley, P. S., Harty, D. W. S., WyatT, J. E., Brown, C. R., Doran, J. P. \& Gibes, A. C. C. (1987). A comparison of the adhesion, coaggregation and cell surface hydrophobicity properties of fibrillar and fimbriate strains of Streptococcus salivarius. Journal of General Microbiology 133, 3207-3217.

Hesketh, L. M., WyatT, J. E. \& Handley, P. S. (1987). Effect of protease on cell surface structure, hydrophobicity and adhesion in tufted strains of Streptococcus sanguis biotypes I and II. Microbios 50, 131-145.
JONES, H. C., ROTH, T. L. \& SANDERS, W. M. (1969). Electron microscope study of a slime layer. Journal of Bacteriology 99, 316-325.

LUFT, J. H. (1971). Ruthenium red and violet I. Chemistry, purification, methods of use for electron microscopy and mechanism of action. Anatomical Record 171, 347-368.

Montague, E. A. \& Knox, K. W. (1968). Antigenic components of the cell wall of Streptococcus salivarius. Joumal of General Microbiology S4, 237-246.

Rogers, H. J., Perkns, H. R. \& WARD, J. B. (1987). Additional polymers in bacterial walls. In Microbial Cell Walls and Membranes, pp. 215-238. London: Chapman \& Hall.

Rosenberg, M., Gutnick, D. \& Rosenberg, E. (1980). Adherence of bacteria to hydrocarbons: a simple method for measuring cell surface hydrophobicity. FEMS Microbiology Letters 9, 29-33.

SHOCKMAN, G. D. \& BARRETT, J. F. (1983). Structure, function and assembly of cell walls of Gram-positive bacteria. Annual Review of Microbiology 37, 501-527.

TzapHIIDOU, M. \& ChapMan, J. A. (1986). A study of staining for electron microscopy using collagen as a model system. IV. Phosphotungstate/tungstate negative staining patterns and their correlation with sequence data. Micron and Microscopica 17, 269-280.

WebrKaMp, A. H. \& Jacons, T. (1982). Cell wallassociated protein antigens of Streptococcus salivarius: purification, properties, and function in adherence. Infection and Immunity 38, 233-242.

WeErKaMP, A. H., HANDley, P. S., BAars, A. \& Slot, J. W. (1986). Negative staining and immunoelectron microscopy of adhesion deficient mutants of Streptococcus salivarius reveal that the adhesive protein antigens are separate classes of cell surface fibril. Journal of Bacteriology 165, 746-755.

Werrkamp, A. H., van der Mel, H. C. \& Slot, J. W. (1987). Relationship of cell surface morphology and composition of Streptococcus salivarius $(\mathbf{K})$ to adherence and hydrophobicity. Infection and Immunity 55, 438-445.

WINER, B. J. (1971). Statistical Principles in Experimental Design, p. 359. New York: McGraw Hill. 